\title{
Study on power transmission line ampacity calculation with nonlinear $\mathrm{AC}$ resistance*
}

\author{
Hai-Jun Xue ${ }^{\mathrm{a}}$ and Xu-Dong Zhang ${ }^{\mathrm{b}}$ \\ School of Energy and Power Engineering, \\ Nanjing University of Science \& Technology, \\ Nanjing, China \\ ${ }^{a}$ E-mail: $1036624180 @ q q . c o m,{ }^{b}$ E-mail: zxdhtg@126.com \\ www.njust.edu.cn
}

\begin{abstract}
As the rapid development of renewable energy, energy sectors have to increase the load transfer capacity of transmission line. In order to calculate the line ampacity accurately, it is necessary to establish a valid mathematical model for line AC resistance. However, the simplified AC resistance model, which is current commonly used, does not take into account the nonlinear impact of current variation. In this paper, an modified AC resistance model regarding current-carrying variation is proposed, and the parameters in the proposed model are estimated by the experiment data. Combining IEEE standard and proposed model, the ampacity of conductor LGJ-120/25 is calculated and analyzed. The calculated results indicates that the ampacity obtained by proposed model is less than ampacity obtained by simplified AC resistance model. That means the ampacity would be overestimated and the security of transmission line would be reduced when the nonlinear impact of current variation is ignored.
\end{abstract}

Keywords: Power Transmission Line; Ac Resistance; Ampacity Calculation; Parameter Estimation.

\section{Introduction}

Renewable energy is characterized by clean, few emissions and harmony with human ecological environment. However, there are transmission and consumptive problems in that the renewable energy bases are far away from the load centers. The load transfer capacity is dictated by special weather conditions and the line maximum allowable temperature, but the actual meteorological factors are incompatible with specific condition. The ampacity will be redetermined by the energy sector according to the actual operating conditions [1]. Therefore, the ampacity calculation has important influence on the safety of actual engineering.

The Joule heat generated by AC resistance is the main element of conductor heating, so that accurate calculation of resistance is of importance in ampacity

\footnotetext{
${ }^{*}$ This work is supported by grant 51506092 of the National Natural Science Fundation.
} 
calculation [2]. The simplified model is usually used to calculate line resistance, and the resistance is simply seen as a linear function of its operating temperature [3]. However, in the actual calculation, line resistance is not only linear with temperature. Especially at high temperature, the simplified model is unable to accurately calculate the resistance. In addition, the research in Reference [4-6] has shown that there is a current-dependent behavior in line $\mathrm{AC}$ resistance. The conductors of transmission line are composed of aluminum strands wound upon a steel core of one or more strands. The current flows spirally around the wire that will produce magnetic flux longitudinally within the core and then generate hysteresis and eddy current losses, thus bringing additional AC resistance increments [7].

In this work, an experiment is performed to measure the line resistance for an overhead conductor LGJ-120/25. Results obtained in the laboratory are applied to establish a modified AC resistance model. And the line ampacity is calculated and analyzed under different atmospheric conditions.

\section{Modified Line AC Resistance Model}

\subsection{Traditional line resistance calculation}

In current engineering calculation, high voltage transmission line resistance is calculated solely as a linear function of conductor temperature which is given by:

$$
R_{\mathrm{AC}}=R_{\mathrm{ref}}\left[1+\alpha\left(T-T_{\text {ref }}\right)\right]
$$

where $T$ and $T_{\text {ref }}$ are the operating temperature and the reference temperature, $R_{\mathrm{AC}}$ the line AC resistance and $R_{\text {ref }}$ the reference resistance at temperature $T_{\text {ref, }}$, and $\alpha$ the temperature coefficient.

\subsection{Resistance measurement}

The AC resistance measurement platform is executed at high tension laboratory depicted in Figure 1. The experimental bench components are: experimental conductor, strong current generator, data acquisition, and PC.

The heating of the conductor is performed through the electrical circuit. This circuit composed by T-connector and electrical conductor LGJ-120/25 with seven aluminum wires and seven steel core reinforcing wires. The strong current generator is mounted on one side of the platform so that the current necessary to do the heating is transferred by magnetic induction. Voltage probes, current transformer and a set of thermocouples are fixed on another side of the platform and connected to a data acquisition. 


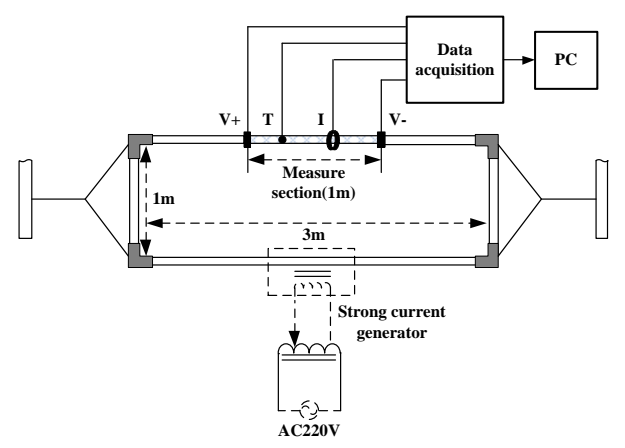

Fig. 1. Experimental platform for AC resistance.

The measurement result of line AC resistance is shown in Figure 2. The resistance increases along with running temperature, while increases at first and then decreases with the increase of current. The above experimental phenomenon shows good agreement with the research of Reference [8], which verifies the nonlinear property of line AC resistance.

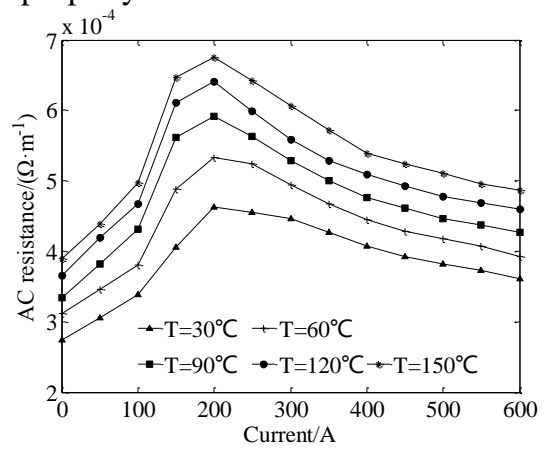

Fig. 2. Experimental results of AC resistance

\subsection{Modified AC resistance model establishment}

The modified AC resistance model presented in this paper can accurately describe the nonlinear characteristic of line resistance, so that it can be used in the ampacity calculation. The modified model is expressed as follows:

$$
R_{A C}=f(T, I)=\sum_{i=0}^{p} \sum_{j=0}^{q} a_{i j} T^{i} I^{j}
$$

where $I$ is the line load, $a_{i j}$ the model parameters, $p$ and $q$ the order of $T$ and I.

The model parameters are evaluated by using least square method. In this work, $p=q=4$, and the valuation results are presented in Table 1 . 
Table 1. Parameters of the modified AC resistance model

\begin{tabular}{cc}
\hline Parameter & Value \\
\hline$a_{00}$ & $5.377 \mathrm{e}-004$ \\
$a_{10}$ & $2.883 \mathrm{e}-005$ \\
$a_{01}$ & $3.312 \mathrm{e}-005$ \\
$a_{20}$ & $-1.531 \mathrm{e}-007$ \\
$a_{11}$ & $9.773 \mathrm{e}-009$ \\
$a_{02}$ & $-1.016 \mathrm{e}-007$ \\
$a_{30}$ & $4.044 \mathrm{e}-010$ \\
$a_{21}$ & $8.693 \mathrm{e}-011$ \\
$a_{12}$ & $-5.712 \mathrm{e}-011$ \\
$a_{03}$ & $9.174 \mathrm{e}-011$ \\
\hline
\end{tabular}

\section{Ampacity Calculation and Analysis}

\subsection{Principle and method}

The heat balance equation is expressed as [3]:

$$
I^{2} R_{A C}+q_{s}=q_{c}+q_{r}
$$

where $q_{s}, q_{c}$ and $q_{r}$ are rate of solar heat gain, convection heat loss rate and radiated heat loss rate.

When the term $\left(R_{\mathrm{AC}}\right)$ is calculated by linear simplified model, the ampacity formula can be obtained by the Eq. (3):

$$
I=\sqrt{\frac{q_{c}+q_{r}-q_{s}}{R_{A C}}}
$$

And when the resistance is calculated using the modified model, with Eq. (2)and (3), the heat balance equation becomes:

$$
\sum_{i=0}^{p} \sum_{j=0}^{q} a_{i j} T^{i} I^{j+2}=q_{c}+q_{r}-q_{s}
$$

the line load $I$ is given by the root of Eq. (5).

\subsection{Analysis of the ampacity calculation result}

Under different weather conditions, the line ampacity is calculated to analyze the differences of results obtained by the simplified model and the modified model. Some conditions such as high ambient temperature of $40^{\circ} \mathrm{C}$, low wind speed of $0.5 \mathrm{~m} / \mathrm{s}$ and strong sunshine about $1000 \mathrm{~W} / \mathrm{m}^{2}$ need to be focused on, since they are considered to be severe conditions affecting the line safety. So in 
the following calculation, just to change one of meteorological parameters, and other parameters are fixed for the worst value.

The calculation results under different environmental temperature and different sunlight intensity are shown in Figure 3, and the line maximum allowable operating temperature is $70^{\circ} \mathrm{C}$. It can be observed that, as the decrease of ambient temperature, the ampacity increases nonlinearly, and the ampacity obtained by the modified model (case a1) is less than the result calculated by the simplified model (case a2). It can also be observed in Figure 3, as the reduction of sunshine intensity, the carrying capacity increases. And on the whole, the current calculated by the formula (5) (case b1) is less than that of formula (4) (case b2).

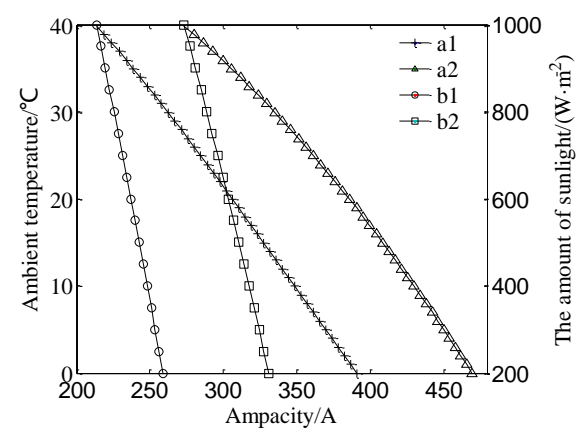

Fig. 3. Ampacity calculation results under different ambient temperature and sunlight intensity.

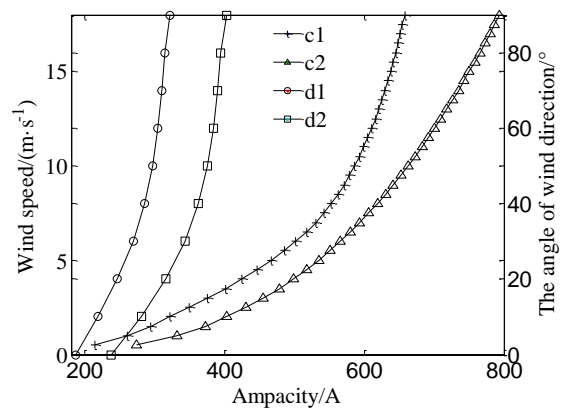

Fig. 4. Ampacity calculation results under different wind speed and wind direction.

In Figure 4, case c1 and case $\mathrm{c} 2$ are the results of the simplified model and the modified model with wind velocity, and the deviations increase gradually with the enhancement of wind speed. Moreover, as the incensement of angle of wind direction, case $\mathrm{d} 1$ obtained by the simplified model and case $\mathrm{d} 2$ calculated by the modified model increase first and then level off, and achieve maximum at the angle of $90^{\circ}$. 


\section{Conclusion}

In this work, an experiment of $\mathrm{AC}$ resistance measurement for an overhead conductor LGJ-120/25 is performed, and the AC resistance correction model is proposed. Then the ampacity of transmission line is calculated and analyzed. A conclusion is brought by the results of experiment that the line AC resistance is not only affected by operating temperature and is also closely related to the current through the line. Furthermore, a comparison of calculation result between the modified model and the simplified model indicates that the environmental meterological factors and the electrical parameters of conductor need to be carefully considered in the ampacity calculation.

\section{References}

1. Crainic E D, Do X D, Meyere P. A fast and exact method for real-time overhead conductor ampacity evaluation[J]. Canadian Electrical Engineering Journal, 1987, 12(3):123-130.

2. Zhang H, Han X S, Wang Y L. Analysis on Current Carrying Capacity of Overhead Lines Being Operated[J]. Power System Technology, 2008.

3. Muratori B D. 738-2012 - IEEE Standard for Calculating the CurrentTemperature Relationship of Bare Overhead Conductors[J]. 2013, 2(2):1-72.

4. Bockarjova, M, Andersson, G. Transmission Line Conductor Temperature Impact on State Estimation Accuracy[J]. Molecular Microbiology, 2008, 68(3):642-656.

5. Morgan V T. The Current Distribution, Resistance and Internal Inductance of Linear Power System Conductors-A Review of Explicit Equations[J]. Power Delivery IEEE Transactions on, 2013, 28(3):1252-1262.

6. Morgan $\mathrm{V}$ T. Electrical characteristics of steel-cored aluminum conductors[J]. Proceedings of the Institution of Electrical Engineers, 1965, 112(2):325-334.

7. Morgan V T, Zhang B, Findlay R D. Effect of magnetic induction in a steelcored conductor on current distribution, resistance and power loss[J]. IEEE Transactions on Power Delivery, 1997, 12(3):1299-1308.

8. Findlay R, Liu F. Experiments on Single Layer ACSR Conductors[C]// Electromagnetic Field Computation, 2006, Biennial IEEE Conference on. 2006:306-306. 